\title{
Juego y actividad física como indicadores de calidad en Educación Infantil Games and physical activity as indicators of quality in Early Childhood Education
}

Francisco Javier Gil-Espinosa, Ángel Ramón Romance García,Adriana Nielsen Rodríguez Universidad de Málaga (España)

\begin{abstract}
Resumen. La etapa de Educación Infantil está directamente relacionada con el desarrollo personal y es crucial en el proceso de crecimiento personal. Las experiencias de aprendizaje no pueden ser desgajadas en áreas diferenciadas de desarrollo cognitivo, social, emocional y físico, sino que se encuentran integradas y son dependientes. Este equilibrio se puede lograr a través del juego creativo e interactivo, que soporta y proporciona andamiaje a todas las áreas de desarrollo y de contenido curricular. Desde esta perspectiva, el presente trabajo realiza una puesta en común entre legislación y conocimiento científico en relación al juego y la actividad física en Educación Infantil finalizando con una propuesta de aplicación práctica. Los objetivos de la investigación son: (I) Revisar el conocimiento científico y legislación en relación a la utilización metodológica del juego en educación infantil, (II) revisar el conocimiento científico y legislación en relación a la actividad física en Educación Infantil y (III) realizar una propuesta de aplicación práctica. Se concluye que el juego y actividad física son dos variables fundamentales para lograr una metodología globalizadora, significativa y motivante que aporte calidad al proceso formativo del alumnado en la etapa de educación infantil que puede complementarse con la utilización de recursos digitales y tecnológicos para el aprendizaje. A su vez, se presentan unas líneas generales de intervención, por áreas y bloques de contenidos, al objeto de mejorar la calidad del proceso de enseñanza-aprendizaje.
\end{abstract}

Palabras clave: Educación infantil, calidad educativa, juego, actividad física.

Abstract. The stage of Early Childhood Education is directly related to personal development and is crucial in the process of personal growth. Learning experiences cannot be split into differentiated areas of cognitive, social, emotional and physical development, however they are integrated and dependent. This balance can be achieved by means of creative and interactive play, which supports and provides scaffolding to all areas of curriculum development and content. From this perspective, the present work brings together legislation and scientific knowledge in relation to play and physical activity in Early Childhood Education, ending with a practical application proposal. The research objectives are: (I) Review scientific knowledge and legislation regarding the methodological use of play in early childhood education; (II) Reviewing scientific knowledge and legislation in relation to physical activity in early childhood education; and (III) Making a practical implementation proposal. It is concluded that play and physical activity are two fundamental variables to achieve a globalizing, meaningful and motivating methodology which provides quality to the students' formative process in childhood education stage which can be complemented with the use of digital and learning technological resources. At the same time, general lines of intervention are provided, in areas and blocks of contents, in order to improve the quality of the teaching-learning process. Key words: Early Childhood education, education quality, play, physical activity.

\section{Introducción}

La etapa de Educación Infantil está directamente relacionada con el desarrollo personal y es crucial en el proceso de crecimiento debido a que las experiencias de la primera infancia pueden influir en todo el ciclo de vida de un individuo (OMS, 2013). A su vez, obviar alguna de las esferas esenciales en el desarrollo humano y su proceso educativo no permitirá alcanzar unos niveles deseables de calidad de vida (Gil \& Aznar, 2016). Por tanto, las experiencias de aprendizaje de los niños pequeños no pueden ser desgajadas en áreas diferenciadas de desarrollo cognitivo, social/emocional y físico, sino que se encuentran integradas y son dependientes. Este equilibrio se puede lograr a través del juego creativo e interactivo, que soporta y proporciona andamiaje a todas las áreas de desarrollo y de contenido curricular (Lindsey, 2013).

La UNESCO (1989), en la Convención sobre los Derechos del Niño, artículo 31, establece: «Los Estados Partes reconocen el derecho de la niñez al descanso y la diversión, a jugar y realizar actividades recreativas apropiadas para la edad, y a participar libremente en la vida artística y cultural». Los niños no solo tienen derecho a jugar, sino que los Estados deben «proveer oportunidades para el desarrollo de actividades culturales, artísticas y recreativas». De hecho, la legislación educativa española contempla la importancia del juego y del movimiento en el desarrollo infantil (Ley Orgánica 2/2006, modificada por la Ley Orgánica 8/2013y Real Decreto 1630/2006), definiendo la Educación Infantil como una etapa educativa con identidad propia que atiende a niñas y niños desde el nacimiento hasta los seis años de edad, con la finalidad de contribuir a su desarrollo físico, afectivo, social e intelectual, y que sienta las bases para el desarrollo personal y social por medio de la integración de aprendizajes que cimentan el posterior desarrollo de competencias consideradas básicas para todo el alumnado. De hecho, el juego, la infancia y actividad física han estado unidos a través de la historia

Fecha recepción: 08-10-17. Fecha de aceptación: 04-02-18 Francisco Javier Gil Espinosa javiergil@uma.es
(Andreu-Cabrera, Cepero, Rojas \& Chinchilla-Mira, 2010).

El presente trabajo realiza una puesta en común entre legislación y conocimiento científico en relación al juego y la actividad física en Educación Infantil finalizando con una propuesta de aplicación práctica.

Los objetivos de la investigación son: (I) Revisar el conocimiento científico y legislación en relación a la utilización metodológica del juego en educación infantil,(II) revisar el conocimiento científico y legislación en relación a la actividad física en Educación Infantil y (III) realizar una propuesta de trabajo utilizando el juego y la actividad física con criterios decalidad.

\section{Método}

Utilizando como referencia los objetivos planteados en el trabajo, se ha realizado una revisión de la legislación educativa española y la literatura científica en relación al juego y la actividad física en Educación Infantil con el propósito de sintetizar los estudios publicados en relación a su importancia en el desarrollo infantil.

No se emplearon parámetros temporales como criterios de exclusión de artículos, ni tampoco hubo restricciones en el criterio de idiomas. El presente trabajo no pretende ser una revisión exhaustiva de la investigación sobre el juego y la actividad física en la etapa de educación infantil, sino un análisis de la importancia que tanto el legislador como la literatura científica otorgan a ambos.

\section{Resultados}

El juego y la actividad física en el sistema educativo español en educación infantil

En lo que respecta a la etapa de Educación Infantil, pocas han sido las modificaciones introducidas por la Ley Orgánica 8/2013, de 9 de diciembre, para la mejora de la calidad educativa (LOMCE, 2013), por lo que habremos de seguir atendiendo al texto (en cualquier caso, al texto consolidado) de la Ley Orgánica 2/2006, de 3 de mayo, de Educación (LOE, 2006), el cual establece en su artículo 14.3, que «en ambos ciclos de la educación infantil se atenderá progresivamente al desarrollo 
afectivo, al movimiento y los hábitos de control corporal,..., así como al descubrimiento de las características físicas y sociales del medio en el que viven. Además, se facilitará que niñas y niños elaboren una imagen de sí mismos positiva y equilibrada y adquieran autonomía personal», y a su vez indica en el artículo 14.6 que «los métodos de trabajo en ambos ciclos se basarán en las experiencias, las actividades y el juego y se aplicarán en un ambiente de afecto y confianza, para potenciar su autoestima e integración social».

Por otra parte, dicha Ley 2/2006 establece en su artículo 13, entre otros objetivos, que la Educación Infantil contribuirá a desarrollar en las niñas y niños las capacidades que les permitan: a) Conocer su propio cuerpo y el de los otros, sus posibilidades de acción y aprender a respetar las diferencias; c) Adquirir progresivamente autonomía en sus actividades habituales; e) Relacionarse con los demás y adquirir progresivamente pautas elementales de convivencia y relación social, así como ejercitarse en la resolución pacífica de conflictos y g) Iniciarse en el movimiento, el gesto y el ritmo.

De esta forma, el currículo se orienta a lograr un desarrollo integral y armónico de la persona en los distintos planos: Físico, motórico, emocional, afectivo, social y cognitivo, y a procurar los aprendizajes que contribuyen y hacen posible dicho desarrollo (Real Decreto 1630 2006, de 29 de diciembre, por el que se establecen las enseñanzas mínimas del segundo ciclo de Educación infantil), estableciendo las siguientes áreas: (1) Conocimiento de sí mismo y autonomía personal, (2) Conocimiento del entorno y (3) Lenguajes: Comunicación y representación.

En el cuadro 1 se describen las características principales de cada área y su relación con el juego y la actividad física, según establece el RD 1630/2006, de 29 de diciembre.

En cualquier caso, no se tiene más que seguir ahondando en la legislación educativa vigente, en los distintos niveles de concreción curricular, para encontrar nuevas alusiones a la importancia del juego en la Educación Infantil, proponiéndose métodos de trabajo basados en las experiencias, las actividades y el juego, así como promover el juego como actividad privilegiada que integra la acción con las emociones y el pensamiento, y favorece el desarrollo social. De forma específica se establece el trabajo para el desarrollo de la «confianza en las propias posibilidades de acción, participación y esfuerzo personal en los juegos y en el ejercicio físico» (Real Decreto 1630/2006, de 29 de diciembre, p. 477).

Entre otros, el currículo de Educación Infantil de la Comunidad Autónoma de Andalucía, desarrollado en su Orden de 5 de agosto de 2008, no sólo contempla todo lo expuesto anteriormente mediante constantes alusiones al juego y a la actividad física, entre las que se incluye la consideración del juego como «recurso básico» gracias al cual el niño y la niña «van ampliando el conocimiento de símismo, avanzando en la construcción de su identidad y de las posibilidades de la relación social» y se concibe la necesidad de incluir en la jornada escolar aquellas «experiencias, juegos y actividades que impliquen movimiento -arrastrar, gatear, caminar, saltar, subir, bajar, correr-- y diferentes posturas del cuerpo -tumbado, sentado, de pie-» con el fin de que los niños y niñas exploren sus posibilidades motrices, adquieran una coordinación dinámica general y alcancen un mayor control del movimiento.

Así, el desarrollo curricular de la Educación Infantil en España, cumple con la carta internacional de la Educación Física y el Deporte que, en su artículo primero, establece que «todo ser humano tiene el derecho fundamental de acceder a la educación física y al deporte, que son indispensables para el pleno desarrollo de su personalidad. El derecho a desarrollar las facultades físicas, intelectuales y morales por medio de la educación física y el deporte deberá garantizar tanto dentro del marco del sistema educativo como en el de los demás aspectos de la vida social» (UNESCO, 1978, p. 33).

\section{El juego en el desarrollo infantil}

Cuando el niño juega, permanece activo. Todo el tiempo hace nuevas observaciones, pregunta y responde, hace elecciones y amplía su imaginación y su creatividad. El juego proporciona al pequeño la estimulación y la actividad física que su cerebro necesita para desarrollarse y poder aprender en el futuro (UNICEF, 2004). El juego es una herramienta de aprendizaje (Dikici, 2016; Linaza, 2013; Paley, 2005; Zych, Ortega-Ruiz \& Sibaja, 2016), de hecho, «existe un fuerte y creciente conjunto de conocimientos que identifica el vínculo entre el juego y el desarrollo de las habilidades cognitivas y sociales que son prerrequisitos para aprender conceptos más complejos a medida que los niños crecen y al identificar la necesidad de pensadores creativos e innovadores» (Lindsey, 2013, p.132).

Por tanto, el juego se constituye como una actividad irremplazable para desarrollar la capacidad de aprendizaje de los niños y niñas, un medio de expresión y de maduración en el plano físico, cognitivo, psicológico y social (Grellet, 2000). Así, por ejemplo, Dikici (2016) encuentra puntuaciones estadísticamente significativas en los niños y niñas que habían realizado un entrenamiento de ajedrez tanto en el pensamiento creativo como en las pruebas de la Teoría de la Mente que, a su vez, están correlacionadas con habilidades cognitivas sociales. Igualmente, Zych et al. (2016) concluyen que el juego infantil es la actividad privilegiada para el desarrollo afectivo que debe estimular la educación.

La evidencia de la investigación deja muy claro que el juego tiene un papel en el desarrollo general de los niños pequeños (Bergen, 2002), 
siendo una parte esencial en el desarrollo porque contribuye al bienestar cognitivo, físico, social y emocional de niños y adolescentes (Durán, López, Fernández, García \& García, 2014).Por otro lado, el aprendizaje basado en el juego y narración de cuentos son métodos para generar motivación intrínseca en el aprendizaje (Laine, Nygren, Dirin \& Suk, 2016). Así, por ejemplo, Tabernero, Aliseda \& Daniel (2016) concluyen tras su experiencia «Jugar a los cuentos» que los participantes (niños y niñas de cinco años) llegan a adquirir aprendizajes globales e interdisciplinares a partir del movimiento.

Sin embargo, «no siempre se aprecia el papel decisivo que el juego desempeña en el desarrollo infantil. Los procesos del juego inûuyen la formación sináptica yestán vinculados con el apego seguro a cuidadores y las relaciones con otros niños» (Irwin, Siddiqi \& Hertzman, 2007, p. 22). La limitada evidencia investigativa vigente sugiere que los educadores deberían resistir las políticas que reduzcan el tiempo para el juego social de aparentar y trabajar para aumentar los fondos para la investigación sobre las relaciones entre el juego y los procesos cognoscitivos en la niñez temprana (Bergen, 2002).

\section{La actividad física en el desarrollo infantil}

La Actividad física (AF) es un término amplio que comprende cualquier movimiento corporal que exija gasto de energía (UNESCO, 2015). La actividad física se puede definir como cualquier movimiento corporal producido por los músculos esqueléticos y con un gasto de energía más allá de lo que puede ocurrir durante las actividades típicas de la vida diaria (USDHHS, 2008). Además de la educación física y el deporte, la AF incluye el juego activo y actividades habituales y cotidianas.

La declaración de Berlín afirma que «la educación física en la escuela yen todas las demás instituciones educativas es el medio más efectivo para dotar a todos los niños y jóvenes de competencias, aptitudes, actitudes, valores, conocimientos y comprensión para su participación en la sociedad a lo largo de la vida» (UNESCO, 2013, p. 3) contribuyendo a la salud y bienestar de los mismos.

En sus Recomendaciones Mundiales sobre Actividad Física para la Salud, la OMS (2010) concluye que, para lograr los beneficios para la salud física y mental, los niños de cinco a 17 años deberían acumular un mínimo de 60 minutos diarios de actividad física moderada o vigorosa. En la misma línea, se recomienda un tiempo real de AF, en horario escolar, entre 180 minutos (UNESCO, 2015) y 225 minutos (National Association for Sport and Physical Education-NASPE-, 2012) semanales avanzando hacia las cinco horas semanas (European Society of Cardiology, 2007; LOMCE, 2013; OMS, 2010).

Los posicionamientos de estos organismos se sustentan en numerosos estudios científicos que encuentran mejoras en el bienestar físico, psíquico y social (Burgess, Grogan \& Burwitz, 2006; Gray \& Leyland, 2008; McIntyre, Chivers, Rose \& Hands, 2014; OMS, 2010; Parlamento Europeo, 2007) producidas por la actividad física. Así, «el movimiento tiene una gran importancia en el desarrollo integral de la infancia» Sugrañes \& Ángels (2008, p. 19), al considerar las autoras que la educación psicomotriz implica aspectos socio-afectivos, motores, psicomotores e intelectuales.

Por otro lado, la actividad y ejercicio físico desempeñan un impor- tante papel sobre la mejora del rendimiento cognitivo y académico de los niños y adolescentes (Ardoy, Fernández-Rodríguez, Jiménez-Pavón, Castillo, Ruiz \& Ortega, 2014; Coe, Pivarnik, Womack, Reeves \& Malina, 2012; González \& Portolés, 2016; Reloba, Chirosa \& Reigal, 2016; Ruiz-Pérez, Navia, Ruiz, Ramón \& Palomo, 2016). Algunas alteraciones del lenguaje infantil están asociadas con determinados déficits neuropsicológicos, como problemas de memoria, de atención, funciones ejecutivas, disfunciones motrices, percepción temporal, reconocimiento táctil, esquema corporal, orientación espacial y discriminación visual (Conde, Conde, Bartolomé \& Quirós, 2009). Así, la actividad física se convierte en una parte de la formación integral de los niños y niñas (De Souza, Posada-Bernal \& Lucio-Tavera, 2017).

Pérez-Pueyo, et al. (2016, p. 65) definen la Competencia Corporal Clave(CCC) como la: «capacidad para utilizar y cuidar nuestro cuerpo de forma adecuada, disfrutary expresarse con él» y Ruiz-Pérez (1995, p.19) la competencia motriz como «el conjunto de conocimientos, procedimientos, actitudes y sentimientos que intervienen en las múltiples interacción que el sujeto realiza en su medio y con los demás, y que permiten que los escolares superen los diferentes problemas motrices planteados tanto en las sesiones de EF como en su vida cotidiana», constituyendo una óptica global de aspectos cognitivos, motores, sociales y emocionales y una más concreta de habilidades motrices específicas (Ruiz-Pérez, 2014), se relacionan directamente con las finalidades educativas, los objetivos, áreas y bloques de contenidos que la legislación española recoge en la etapa de educación infantil.

La competencia corporal o motriz, por tanto, debería llegar a todos los niños, niñas y jóvenes en igualdad de condiciones independientemente del nivel económico, cultural y socio-educativo de su familia, siendo por ello esencial su presencia en el sistema educativo curricular motivado por cuestiones como la prevención de enfermedades, promoción del bienestar a lo largo de la vida, proporción de oportunidades únicas de actividades, mejora de la autonomía y habilidades motoras, promoción del aprendizaje y ayuda a una educación integral (Le Mausier \& Corbin, 2006).

Ello orienta hacia la necesidad de ofrecer experiencias de aprendizaje a los niños a través de actividades físicas que sean apropiadas para ayudarles a adquirir las habilidades psicomotrices, la comprensión cognitiva y las aptitudes sociales y emocionales que necesitan para llevar una vida físicamente activa (UNESCO, 2015 y NASPE, 2012). Así, Lagardera \& Maciano (2014) indican que la trascendencia social de la educación física estriba en la influencia que puede ejercer en la calidad de vida de las personas independientemente de su edad y condición.

Calidad del juego y la actividad física en educación infantil. Propuestas de actuación.

La revisión realizada no deja lugar a dudas sobre el papel protagonista que deben tener la actividad física y el juego en la educación infantil. Sin embargo, encontramos diversos términos como actividad física, educación física, motricidad y psicomotricidad. Por coherencia con el resto de niveles del sistema educativo consideramos conveniente que se asuma el término de «Educación Física», ya que «el movimiento no solo implica acciones motrices, sino cognitivas y afectivas» (Torres, 2015, p. 3). A su vez, como podemos comprobar en el cuadro 2, los contenidos

Cuadro 2

\begin{tabular}{|c|c|c|}
\hline \multirow[t]{3}{*}{ Capacidades perceptivo-motrices } & La percepción corporal & $\begin{array}{l}\text { Imagen y conocimiento corporal, actitud tónico postural equilibradora, relajación, respiración, lateralidad, } \\
\text { sensopercepción. }\end{array}$ \\
\hline & La percepción espacial & La organización espacial \\
\hline & La percepción temporal & La orientación temporal, la estructuración temporal, el ritmo. \\
\hline \multirow[t]{2}{*}{ Habilidades motrices } & Habilidades motrices básicas & Desplazamientos, saltos, giros, lanzamientos y recepciones \\
\hline & Habilidades motrices genéricas & Botes, golpeos, conducciones, fintas, paradas, interceptaciones \\
\hline \multirow[t]{2}{*}{ Capacidades coordinativas } & Coordinación dinámica general & Movimiento coordinado de todo el cuerpo \\
\hline & Coordinación segmentaria & Óculo-manual, óculo-pédica y óculo cefálico \\
\hline Desarrollo afectivo-social (socioafectivo) & \multicolumn{2}{|c|}{$\begin{array}{l}\text { Actividades de grupo, hábitos de higiene, lenguaje corporal, autonomía, juegos y danzas. } \\
\text { Posibilidades de manifestación de iniciativa, improvisación, motivación, control de las emociones y estados de ánimo, autoestima, tolerancia la frustración, } \\
\text { capacidad de espera, aceptación y respeto a los demás. }\end{array}$} \\
\hline Desarrollo cognitivo o mental & \multicolumn{2}{|c|}{$\begin{array}{l}\text { Ritmos y movimientos lineales para reforzar la lectoescritura. } \\
\text { Desarrollo de la capacidad de recepción de estímulos } \\
\text { Memorizar consignas, recuerdo de hechos, comparaciones. } \\
\text { El descanso y la relajación como espacio para organizar el pensamiento. } \\
\text { Representación mental y simbolización como inicio de la abstracción. } \\
\text { Establecimiento de relaciones oportunas entre los acontecimientos. }\end{array}$} \\
\hline
\end{tabular}


a desarrollar en EF en la EI están directamente relacionados con áreas y bloques de contenidos que contempla el RD 1630/2006 (cuadro 1).

Aprovechar las posibilidades del juego en su totalidad implica ser conscientes de que el juego debe perseguir un objetivo, que se ejecuta por algo y que debe estar planificado y pensado (Torres, 2015). Para lograr una aplicación de calidad en la utilización del juego, bien como contenido de aprendizaje o como estrategia motivadora (Ruiz \& García, 2004) deberíamos relacionar los juegos con los contenidos de aprendizaje, áreas y bloques de contenidos curriculares, con objetivos motrices, afectivos y sociales.

A su vez, la utilización del juego como actividad de aprendizaje no debe olvidar principios básicos para su organización, planificación, creación y puesta en práctica: Enfoque globalizador y significativo, placentero, voluntario, autotélico, participación activa, integrador y con atención a la diversidad. Por su parte, «uno de los retos a los que deberíamos de dar respuesta sería el llevar a la práctica, en la medida de lo posible, las aportaciones de los pequeños, de tal modo que la investigación no se limite a un mero acer-camiento a conocer lo que ellos quieren sino, también, en hacerles sentir que han sido tenidos en cuenta. Otro reto indudable pasaría por contemplar no solo la mirada individual de cada niño, sino por consensuar la perspec-tiva colectiva mantenida en torno a un determinado foco de interés común, ya que el no hacerlo provocaría el peligro potencial de caer en una perspec-tiva limitada de la participación» (Castro, Ezquerra \& Argos, 2016, p. 120). Resulta especialmente interesante el uso de las nuevas tecnologías y sus beneficios pedagógicos, los cuales «dependerán de la capacidad de los docentes para aprovechar el potencial de este recurso» (Tirado-Morueta \& Aguaded-Gómez, 2014, p. 251), pues «pueden facilitar el desarrollo de estructuras básicas para el pensamiento, como son la atención, la memoria, la organización perceptiva, el lenguaje, la capacidad de análisis...» (García, Escarranza \& Mancebo, 1994, p. 96). De hecho, el profesorado de educación infantil es consciente y valora los beneficios de las tecnologías de la información y comunicación en sus programaciones de aula y de centro (Sevillano \& Rodríguez, 2013). A su vez, es posible utilizar didáctica y lúdicamente los medios de comunicación (Tejero, 1993).

En los cuadros 3, 4 y 5 se presentan los indicadores metodológicos clave de referencia para diseñar actividades lúdico-motrices organizadas por áreas y bloques de contenidos, así como su justificación en base a la literatura científica (Dikiti, 2016; Durán et al., 2014; Gupta, 2009; Gutiérrez, 2003; Lillard, 1998, Orden de 5 de agosto de 2008, Sugrañes \& Ángels, 2008 y Zhang et al., 2016). Estas propuestas de actuación deberían ser reforzadas con la utilización de tecnologías para el aprendizaje y considerar que la educación física en la educación infantil se dirige al desarrollo global pudiéndose organizar en factores perceptivo-motores, físico-motores y afectivos-relacionales (Gil, Contreras \& Gómez, 2008).

La aplicación de una metodología basada en actividades lúdicas y motrices, como la presentada en los cuadros 3,4 y 5 , hace pensar en la necesidad de un profesorado con formación específica al respecto, así, True et al. (2017) concluyen que la formación del profesorado es una variable que afecta a la competencia motriz, entendiendo por esta el

Cuadro 3

\begin{tabular}{|c|c|c|c|}
\hline \multirow{2}{*}{\begin{tabular}{l}
\multicolumn{1}{c}{ Área } \\
Conocimiento de sí \\
mismo y \\
autonomía \\
personal
\end{tabular}} & \multicolumn{2}{|c|}{ Bloques } & Propuesta de actuación \\
\hline & \multicolumn{2}{|c|}{ 1. El cuerpo y la propia imagen } & $\begin{array}{l}\text { Juegos de imitación (reproducir gestos, expresiones faciales y sonidos, imitación de animales y objetos en movimiento), juegos de grupo y } \\
\text { con tareas compartidas, juegos delante del espejo, la identificación con su nombre, el reconocimiento de sus objetos personales, la } \\
\text { identificación con imágenes, o símbolos que señalan sus pertenencias, juegos de oposición, de diferenciación con los otros. }\end{array}$ \\
\hline & \multicolumn{2}{|l|}{ 2. Juego y movimiento } & $\begin{array}{l}\text { Juegos y actividades que impliquen movimiento -arrastrar, gatear, caminar, saltar, subir, bajar, correr- y diferentes posturas del cuerpo - } \\
\text { tumbado, sentado, de pie-, podrán explorar sus posibilidades. Utilizar objetos de distinto peso, volumen, tamaño, colores, formas y } \\
\text { texturas, } \\
\text { Rebotar, sonar, apretar, arrugar, encajar, enroscar, modelar, rasgar, lanzar, mover, ... }\end{array}$ \\
\hline & \multirow{2}{*}{\multicolumn{2}{|c|}{$\begin{array}{l}\text { 3. La actividad y la vida cotidiana } \\
\text { 4.El cuidado personal y la salud }\end{array}$}} & Situaciones de contacto personal con sus iguales, en tareas compartidas y en los juegos de grupo. \\
\hline & & & Juegos de imitación de actividades saludables y de cuidado personal. \\
\hline \multicolumn{4}{|l|}{ Cuadro 4.} \\
\hline \multirow{4}{*}{$\begin{array}{l}\text { Área } \\
\text { Conocimiento del } \\
\text { entorno }\end{array}$} & Bloques & & Propuesta de actuación \\
\hline & $\begin{array}{l}\text { 1. Medio físico: } \\
\text { Elementos, relaciones y } \\
\text { medida }\end{array}$ & $\begin{array}{l}\text { Vivenci } \\
\text { rodar). } \\
\text { permeal } \\
\text { Activid } \\
\text { Cancior } \\
\text { Contact } \\
\text { Especia } \\
\text { Juegos } \\
\text { Número }\end{array}$ & $\begin{array}{l}\text { partir y experimentar con objetos y elementos del medio físico a través del movimiento y manipulación (desplazar, tirar, arrastrar, hacer } \\
\text { rimiento de algunas de sus características y propiedades: sabor, color, forma, peso, tamaño, textura, ductilidad, plasticidad, fluidez, dureza, } \\
\text { etc. } \\
\text { imitación y de juegos simbólicos para la observación y uso de esos objetos en nuestro medio. } \\
\text { titivas, cantos rítmicos, cuentos y poemas. } \\
1 \text { agua, la arena, etc. La utilización de instrumentos de medida reales como balanzas, metros y vasos medidores. } \\
\text { tancia se dará a la realización de desplazamientos orientados a través de juegos y vivencias cotidianas: desplazarse desde, hacia, hasta, etc. } \\
\text { namiento (ajedrez...) } \\
\text { rales y manuales }\end{array}$ \\
\hline & $\begin{array}{l}\text { 2. Acercamiento a la } \\
\text { naturaleza }\end{array}$ & $\begin{array}{l}\text { Inclusió } \\
\text { diferent } \\
\text { Moviliz } \\
\text { Ofrecer } \\
\text { rincón c }\end{array}$ & $\begin{array}{l}\text { j juegos y narraciones ilustradas con imá́genes y sonido los elementos de la naturaleza (animales, plantas, piedras, arena, agua...). Incorporar } \\
\text { extos -campo, mar, montañas, selva, desierto- } \\
\text { ipótesis y teorías que niños y niñas se formulan sobre como suceden las cosas. } \\
\text { nidades de entrar en contacto directo con el medio natural: salidas al campo, cuidado de animales en el aula, pequeñas experiencias en el } \\
\text { aleza, cultivo de un huerto escolar, etc. }\end{array}$ \\
\hline & $\begin{array}{l}\text { 3. Cultura y vida en } \\
\text { sociedad }\end{array}$ & $\begin{array}{l}\text { Activid } \\
\text { confecc } \\
\text { Incorpo }\end{array}$ & $\begin{array}{l}\text { e provoquen la toma de conciencia y reflexión sobre las diversas experiencias de relación social -taller de disfraces, día de la paz, } \\
\text { un regalo para un compañero que está enfermo, ... } \\
\text { orogresiva de pautas adecuadas de comportamiento social a través de cuentos, juegos de roles y de imitación. }\end{array}$ \\
\hline
\end{tabular}

Cuadro 5.

Propuesta de actuación en relación al área de Lenguajes: Comunicación y representación.

\begin{tabular}{|c|c|c|}
\hline Área & Bloques & Propuesta de actuación \\
\hline \multirow[t]{4}{*}{$\begin{array}{l}\text { Lenguajes: } \\
\text { Comunicación y } \\
\text { representación }\end{array}$} & 1. Lenguaje verbal & $\begin{array}{l}\text { Situaciones de escucha y comprensión de cuentos, relatos, leyendas, poesías, adivinanzas, trabalenguas, etc., tradicionales o contemporáneas. } \\
\text { Juegos de roles y representación teatral. } \\
\text { Participación activa en juegos de palabras - espontáneos o dirigidos -. } \\
\text { Situaciones donde los niños y niñas escuchen, para comprender globalmente, fragmentos de canciones, cuentos, poesía o rimas en lengua } \\
\text { extranjera. } \\
\text { Abecedario corporal y manual. }\end{array}$ \\
\hline & $\begin{array}{l}\text { 2. Lenguaje audiovisual y tecnologías } \\
\text { de la información y la comunicación }\end{array}$ & $\begin{array}{l}\text { Jugar con la luz y el color, la opacidad y la transparencia, descubrir cómo crear el movimiento en imágenes, etc., desarrollando capacidades } \\
\text { vinculadas a la observación y aprecio artístico de la imagen. } \\
\text { Utilización de medios como ordenador, periféricos, cámara digital, reproductores de audio y vídeo, consolas de juego, móviles, etc., permitirá a } \\
\text { los niños y niñas ir conociendo e interpretando los distintos lenguajes de las tecnologías. } \\
\text { Video juegos activos (con actividad física involucrada). }\end{array}$ \\
\hline & 3. Lenguaje artístico & $\begin{array}{l}\text { Manifestaciones, acompañadas de movimientos, balanceos, palmadas, golpecitos, gestos, etc. Interactuando con objetos sonoros. } \\
\text { Las canciones de corro, juegos, cantinelas, cancioncillas populares. } \\
\text { Participación en juegos y actividades sobre el reconocimiento, imitación y reproducción de ritmos, melodías movimientos y sonidos del entorno } \\
\text { natural y social. Participación en situaciones colectivas donde se haga necesario la interpretación y memorización de canciones, en } \\
\text { dramatizaciones. Canciones de corro, juegos, cantinelas, cancioncillas populares. } \\
\text { Actividades de dibujo diversas -ilustración de cuentos, comunicar información, representar una situación de la realidad-, donde se empleen útiles } \\
\text { y soportes diversos. } \\
\text { Actividades motrices con las manos. }\end{array}$ \\
\hline & 4. Lenguaje corporal & $\begin{array}{l}\text { La participación en juegos de imitación, -de personas, animales, objetos o situaciones-, en las actividades de la vida cotidiana, así como la } \\
\text { representación de personajes, hechos y situaciones mediante juegos simbólicos, disfrutando de dramatizaciones y, en otros juegos de expresión } \\
\text { corporal. } \\
\text { Expresiones y comunicación de ideas, sensaciones y sentimientos, así como la utilización de la danza y el baile } \\
\text { La dramatización y el juego dramático con utilización del lenguaje corporal. } \\
\text { Actividades lúdicas de comunicación con el cuerpo y las manos y actividades lúdicas de comunicación de carácter vivencial (imagen, gesto, } \\
\text { sonido, olfato... }\end{array}$ \\
\hline
\end{tabular}


nivel para ejecutar habilidades motrices básicas.

\section{Conclusiones}

La importancia del juegoy la actividad física en el desarrollo infantil sugiere la necesidad de incorporar profesorado especialista en Educación Física en dicha etapa con la intencionalidad de mejorar el desarrollo integral del alumnado y garantizar la utilización del tiempo necesario en horario escolar y curricular.

\section{Futuras líneas de investigación}

Al no contemplar la legislación en Educación Infantil un horario específico para Educación Física ni requerir una formación específica del profesorado, sería conveniente un análisis del tiempo dedicado a la actividad física en el desarrollo curriculary su relación con las características y formación del profesorado en España.

\section{Referencias}

Andreu-Cabrera E., Cepero M., Rojas F.J., Chinchilla-Mira J.J. (2010). Play and childhood in ancient Greece. J. Hum. Sport Exerc.5(3), 339-347.

Ardoy, D.M., Fernández-Rodríguez, J.M., Jiménez-Pavón; D., Castillo, R., Ruiz, J.R \& Ortega, F.B. (2014). A Physical Education trial improves adolescents' cognitive performance and academic achievement: the EDUFIT study. Scandinavian Journal of Medicine \& Science in Sports,24, 52-61. doi:10.1111/sms.12093

Aznar, P., Morte, J.L., Serrano, R. \& Torralba, J. (1998). La Educación física en la Educación infantil de 3 a 6 años. Barcelona: Inde. 15120.

Bergen, D. (2002). El juego de aparentar y el desarrollo de los niños pequeños. Clearing house on Elementary and Early Childhood Education. 1-2. Recuperado de https://www.researchgate.net/ publication/255664973

Burgess, G., Grogan, S., \& Burwitz, L. (2006). Effects of a 6-week aerobic dance intervention on body image and physical selfperceptions in adolescent girls. BodyImage, 3(1), 57-66. doi: http:/ /dx.doi.org/10.1016/j.bodyim.2005.10.005

Castro, A., Ezquerra, P. \& Argos J. (2016). Procesos de escucha y participación de los niños en el marco de la educación infantil: Una revisión de la investigación. Educación XX1, 19(2), 105-126, doi: 10.5944/educXX1.14271

Coe, D., Pivarnik, J.M., Womack, C.J., Reeves, M.J. \& Malina, R.M. (2012). Health-related fitness and academic achievement in middle school students. The journal of Sports Medicine and Physical Fitness, 52(6), 654-660. Recuperado de http:// www.minervamedica.it/en/journals/sports-med-physical-fitness/ article.php?cod=R40Y2012N06A0654

Conde P.A., Conde, M.J., Bartolomé, M.T. \& Quirós P. (2009). Neuropsychological profiles associated with the children's oral language disorders. Revista de neurología, 48(1), 32-8.

De Souza, M., Posada-Bernal. S. \& Lucio-Tavera, P. (2017). Physical Education in the Early Childhood:A Perspective of Investigation in Education from the Neuroscience. Systemics, cybernetics and informatics, 15(4), 22-25

Dikici,A. (2016).An investigation on the effectiveness of chess training on creativity and theory of mind development at early childhood. Educational Research and Reviews, 11(11), 1056-1063 DOI: 10.5897/ERR2016.2676

Durán, M., López A., Fernández, J. C, García, M. \& García, S. (2014). Estudio piloto sobre la implementación de un programa lúdico en el contexto escolar para la estimulación del lenguaje. Revista de Logopedia, Foniatría y Audiología. 34,171-179. Recuperado de https://iltemprana.files.wordpress.com/2014/01/durc3a1n-y-otros2014-programa-lc3badico-en-estimulacic3b3n-del-lenguaje.pdf

European Society of Cardiology (2007). Carta Europea sobre la salud cardiovascular. Recuperado de http://www.heartcharter.org/ default.aspx

García, C., Escarranza, I. \& Mancebo, P. (1994). Incorporación de los medios de comunicación en educación infantil. Comunicar, 3, 9496.

Gil, F.J. \& Aznar, M. (2016). Educación física de calidad en el sistema educativo español. Revista Española de Educación Física y Deportes, 414, 69-82. Recuperado dehttp://www.reefd.es/index.php/reefd/ article/viewFile/481/467

Gil, P., Contreras, O. \& Gómez, I. (2008). Habilidades motrices en la infancia y su desarrollo desde una educación física animada. Revista Iberoamericana de Educación. 47. 71-96.

Gray, L. \& Leyland, A. (2008). Overweight status and psychological wellbeing in adolescent boys and girls: a multilevel analysis. European Journal of Public Health, 18(6), 616-621. Recuperado de http://eprints.gla.ac.uk/82754/1/82754.pdf

Grellet, C. (2000). El juego entre el nacimiento y los siete años: un manual para ludotecarias. UNESCO Sector de Educación Monografía 14. Recuperado de http://unesdoc.unesco.org/images/0012/ 001213/121323So.pdf

González, J. \& Portolés, A. (2016). Recomendaciones de actividad física y su relación con el rendimiento académico en adolescentes de la Región de Murcia. RETOS, 29, 100-104. Recuperado de http:// recyt.fecyt.es/index.php/retos/article/view/39936/23471

Gutiérrez, M. (2003). Actividades sensorio motrices para la lectoescritura. Ed. Inde. Barcelona. 10-90.

Irwin, L. G, Siddiqi, A. \& Hertzman, C. (2007). Desarrollo de la Primera Infancia: Un Potente Ecualizador. Informe Final. Recuperado de http://www.who.int/social_determinants/publications/ early_child_dev_ecdkn_es.pdf

Lagardera, F. \& Masciano, A. (2014). Un rayo de luz en el lado oculto de la Educación Física. Revista Iberoamericana de Ciencias de la Actividad Física y el Deporte, 31(1), 34-45. Recuperado de https:/ /riuma.uma.es/xmlui/handle/10630/7548

Laine, T.H., Nygren, E., Dirin, A. \& Suk, H.J. (2016). Science Spots AR: a platform for science learning games with augmented reality, Education Tech Research Dev. 64, 507-531. doi:10.1007/s11423015-9419-0

Le Mausier, G. \& Corbin, Ch. (2006). Top 10 Reasons for Quality Physical Education. Journal of Physical Education, Recreation and Dance (JOPERD), 77(6), 44-53. Recuperado de http:// files.eric.ed.gov/fulltext/EJ794467.pdf

Ley Orgánica 2/2006, de 3 de mayo, deEducación (LOE). Recuperada de https://www.boe.es/buscar/pdf/2006/BOE-A-2006-7899consolidado.pdf

Ley Orgánica 8/2013, de 9 de diciembre, para la mejora de la calidad educativa (LOMCE) https://www.boe.es/diario_boe/ txt.php?id=BOE-A-2013-12886

Lillard,A. S. (1998). Playing with a theory of mind. En O. N. Saracho y B. Spodek (Eds.), multiple perspectives on play in early childhood. Albany: State University of New York Press. 11-33.

Linaza, J.L (2013). El juego es un derecho y una necesidad de la infancia. Bordón 65.1. 103-117.

Lindsey, H. (2013). Play and creativity at the center of curriculum and assessment: a New York city school's journey to re-think curricular pedagogy. Bordón 65 (1), 131-146

McIntyre, F., Chivers, P., Rose, E., \& Hands, B. (2014). Exercise can improve physical self-perceptions in adolescents with low motor competence. Human Movement Science, Early View(Online First). 333-343. http://doi.org/10.1016/j.humov.2014.12.003

National Association for Sport and Physical Education (NASPE) y American Heart Association (AHA) (2012). Shape of the Nation Report: Status of Physical Education in the USA. Recuperado de http://www.shapeamerica.org/advocacy/son/2012/upload/2012Shape-of-Nation-full-report-web.pdf

Organización Mundial de Salud (2010). Recomendaciones Mundiales sobre Actividad Física para la Salud. Recuperado de http:// 
apps.who.int/iris/bitstream/10665/44441/1/ 9789243599977_spa.pdf

OMS (2013) El desarrollo del niño en la primera infancia y la discapacidad: Un documento de debate. Recuperado de https:// www.unicef.org/earlychildhood/files/ECDD_SPANISHFINAL_(low_res).pdf

Orden de 5 de agosto de 2008, por la que se desarrolla el Currículo correspondiente a la Educación Infantil en Andalucía. Recuperado dehttp://www.juntadeandalucia.es/boja/2008/169/3

Paley, V. G. (2005). A child's work: The importance of fantasy play. Chicago: University of Chicago Press. 70-95.

Parlamento Europeo (2007). Propuesta sobre la función del deporte en la educación. Resolución sobre la Función del Deporte en la Educación 2007. Recuperado de http://www.europarl.europa.eu/sides/ getDoc.do?pubRef=-//EP//TEXT+REPORT+A6-20070415+0+DOC+XML+V0//ES

Pérez-Pueyo, A., García, O., Hortigüela, D., Aznar, M. \& Vidal, S. (2016). ¿Es posible una (verdadera) competencia clave relacionada con lo motriz? La competencia corporal. Revista española de educación física y deportes, 415, 51-71.

Real Decreto 1630/2006, de 29 de diciembre, por el que se establecen las enseñanzas mínimas del segundo ciclo de Educación infantil. BOE no 4. Recuperado de https://www.boe.es/boe/dias/2007/01/04/pdfs/ A00474-00482.pdf

Reloba, S., Chirosa, L.J. \& Reigal, R.E. (2016). Relación entre actividad física, procesos cognitivos y rendimiento académico de escolares: revisión de la literatura actual. Revista Andaluza de Medicina del Deporte, 9(4):166-172

Ruiz, F. \& García, M.E. (2004) El juego en los contextos escolares de educación infantil y primaria. En: Ruiz, F. y García, ME. Desarrollo de la motricidad a través del juego. Madrid. Gymnos, p. 73-99.

Ruiz-Pérez, L.M. (1995). Competencia motriz. Madrid: Gymnos. 19

Ruiz-Pérez, L.M. (2014). De quéhablamos cuando hablamos de Competencia Motriz. Acción motriz, 12, 37-44. Recuperado de http:// www.accionmotriz.com/autores_ver.php?id=111

Ruiz-Pérez, L.M., Navia, J.A., Ruiz, A., Ramón, I. \& Palomo I. (2016) Coordinación motriz y rendimiento académico en adolescentes. Retos, 29, 86-89. Recuperado dehttp://recyt.fecyt.es/index.php/ retos/article/view/38769

Sevillano, Mª L. \& Rodríguez, R. (2013). Integración de tecnologías de la información y comunicación en educación infantil en Navarra (España). Píxel-Bit. Revista de Medios y Educación, 42. 75-87.

Sugrañes, E. \& Ángels, M. (Coord.) (2008). La educación psicomotriz (3-8 años). Cuerpo, movimiento, percepción, afectividad: una pro- puesta teórico-práctica. Barcelona: Grao. 19-84.

Tabernero, B.,Aliseda, B. \& Daniel, M.J. (2016). ¿Jugamos a los cuentos? Una propuesta práctica de animación a la lectura a través de la Educación Física. Retos, 29, 216-222.

Tejero, R. (1993). Prensa y creatividad en educación infantil. Comunicar, 1, 85-86.

Tirado-Morueta, R. \& Aguaded-Gómez, J. I. (2014). Influencias de las creencias del profesorado sobre el uso de la tecnología en el aula. Revista de Educación, 363, 230-255. DOI: 10.4438/1988-592XRE-2012-363-179

Torres, G. (2015). Enseñanza y aprendizaje de la Educación Física en Educación Infantil. Madrid: Paraninfo. 3-58.

True, L., Pfeiffer, K.A, Dowda, M., Williams, H.G, Brown, W.H., O’Neill, J.R \&. Pate R. (2017). Motor competence and characteristics within the preschool environment. J Sci Med Sport. 20(8):751-755. doi: 10.1016/j.jsams.2016.11.019

UNESCO (1978). Carta internacional de la Educación Física y el Deporte. Recuperada http://portal.unesco.org/es/ev.phpURL_ID=13150\&URL_DO=DO_TOPIC\&URL_SECTION=201.html

UNESCO (1989). Convención sobre los derechos del niño. Recuperado dehttps://www.msssi.gob.es/ciudadanos/proteccionSalud/infancia/DchosInfancia/docs/DerechosNino.pdf

UNESCO (2013). Declaración de Berlín. Quinta Conferencia Internacional de Ministros y Altos funcionarios encargados de la educación física y el deporte. Recuperado de http://unesdoc.unesco.org/ images/0022/002211/221114s.pdf

UNESCO (2015). Educación Física de Calidad. Guía para los responsables políticos. Recuperado de http://unesdoc.unesco.org/images/ 0023/002313/231340s.pdf

UNICEF (2004). Deporte, Recreación y Juego. Recuperado de https:/ /www.un.org/sport/sites/www.un.org.sportfiles/ckfiles/files/200404_UNICEF_Deporte_recreacion_y_juego.pdf

U. S. Department of Health and Human Services-USDHHS- (2008). Physical Activity Guidelines for Americans. Hyattsville, MD: U. S. Dept. of Health and Human Services.

Zhang, T., Moore, W., Gu, X., TszLun, T. \& Gao, Z. (2016). Promoting Children's Physical Activity in Physical Education: The Role of Active Video Gaming. Journal of Teaching, Research, and Media in Kinesiology 1(1), 13.

Zych, I., Ortega-Ruiz, R. \& Sibaja, S. (2016) Children's play and affective development: affect, school adjustment and learning in preschoolers. Infancia y Aprendizaje39(2), 380-400, DOI: 10.1080/ 02103702.2016.1138718

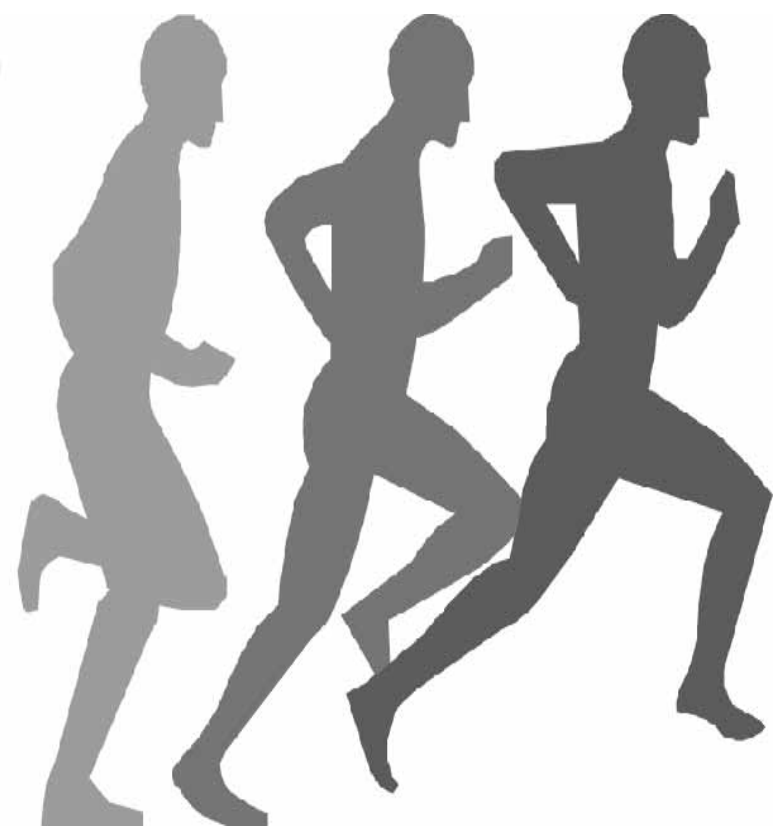

